\title{
Prophylactic role of curcumin against cyclosporine-induced nephrotoxicity: Histological and immunohistological study
}

\author{
Eman A. Abdel Fattah ${ }^{1}$, Hala E. Hashem¹, Fayza A. Ahmed ${ }^{1}$, Mohammed A. Ghallab², \\ Ivan $\operatorname{Varga}^{3}$ and Stefan Polak ${ }^{3}$ \\ ${ }^{1}$ Department of Histology and Cell Biology, Faculty of Medicine, Zagazig University, Zagazig City, Egypt \\ ${ }^{2}$ University Hospital of North Staffordshire, North Staffordshire Royal Infirmary, Princes Road, Stoke-on-Trent, Stafford- \\ shire, ST4 7LN, England \\ ${ }^{3}$ Department of Histology and Embryology, Faculty of Medicine, Comenius University, Sasinkova 4, 81108 Bratislava, \\ Slovakia
}

\begin{abstract}
This study aimed to investigate the possible protective role of curcumin against renal damage caused by administration of cyclosporine A (CsA) in adult male rats. For this purpose, 27 adult male albino rats were used and divided into three equal groups. Group I (control group) and group II (CsA-treated group) received a daily subcutaneous injection of CsA at a dose of $20 \mathrm{mg} / \mathrm{kg}$ b.w. Group III (prophylactic group) received a daily oral curcumin at a dose of $15 \mathrm{mg} / \mathrm{kg}$ b.w. simultaneously with CsA. After 21 days, all the animals were anaesthetized and the kidneys were rapidly removed and processed to prepare paraffin sections stained with H\&E, PAS and Masson's trichrome. In addition, the glutathione S-transferase (GST) enzyme was detected immunohistochemically. The optical density and the area (in \%) of positive GST immunoreactions were measured in the cytoplasm of renal tubules and glomeruli and the data were statistically analyzed. Examination of sections from CsA-treated group showed renal tubules with vacuolated cytoplasm and others with darkly stained pyknotic nuclei. Apical brush borders of proximal tubules were undefined and PAS positive granules were noticed in their cytoplasm. The renal corpuscles contained shrunken glomeruli with widening of their Bowman's spaces. Inflammatory cellular infiltrate and increase in the collagen fibers were observed between the renal tubules. In prophylactic group, the structure of renal tubules and corpuscles were preserved except few tubular darkly stained pyknotic nuclei. Numerous blood vessels, few cellular infiltration and thin collagen fibers were observed between the renal tubules. Statistical analysis of morphometric data showed significant increase in the optical density of GST immunoreactivity in the cells of renal tubules and glomeruli of CsAtreated group when compared with the control or prophylactic groups. However, a significant decrease in the area of GST immunoreactivity in sections from prophylactic group was observed when compared with control or CsA-treated groups. In conclusion, protective effect of curcumin against cyclosporine-induced nephrotoxicity in rats was proven based on the study of histological changes and GST immunoexpression. This study supposes the possible therapeutic applications of curcumin in CsA-treated patients.
\end{abstract}

Key words: Cyclosporine - Glutathione-S-transferase - Curcumin - Renal tissue - Rat

Correspondence to: Stefan Polak, Head of Department of Histology and Embryology, Faculty of Medicine, Comenius University, Sasinkova 4, 81108 Bratislava, Slovakia

E-mail: stefan.polak@fmed.uniba.sk 


\section{Introduction}

Cyclosporine A (CsA) is a cyclic peptide of fungal origin. It is a potent immunosuppressive drug that has been used in organ transplantation and in the management of a number of chronic inflammatory conditions, such as psoriasis, Crohn's disease and rheumatoid arthritis (Faulds et al. 1993). Ying et al. (2003) stated that patients must take this drug over their lifetime. Previous studies reported that a major limiting factor in the clinical use of CsA is chronic nephrotoxicity (Amore et al. 2000; Han et al. 2006). Zhong et al. (2001) and Tirkey et al. (2005) suggested a role for reactive oxygen metabolites as one of the postulated mechanisms in the pathogenesis of cyclosporine nephrotoxicity.

Free radicals and other oxygen-derived species are constantly generated in vivo and cause damage to DNA, lipids, proteins, and other biomolecules. Normally, tissues have defense antioxidant mechanisms that protect them against the toxic effects of free oxygen radicals (Sivonová et al. 2004, 2006; Weis et al. 2005; Kopáni et al. 2006). Glutathione is considered to be the most important watersoluble antioxidant within cells which is critical to normal cellular physiological processes (Rahman and MacNee 1999). The glutathione $S$-transferases (GSTs) are a family of multifunctional cytosolic and membrane-associated microsomal proteins. They can catalyze the conjugation of many electrophilic endogenous and xenobiotic compounds to glutathione (Park et al. 2001). The resultant glutathione conjugates are less toxic than the parent compounds (Greulich et al. 2002).

Curcumin is responsible for the biological actions of turmeric. It has been used as a food additive in curry and in indigenous medicine for centuries. Current traditional indian medicine claims the use of its powder against billiary disorders, anorexia, cough, diabetic wounds, hepatic disorders, rheumatism and sinusitis (Tirkey et al. 2005). Previous studies have shown that curcumin is a natural nontoxic antioxidant possessing a variety of pharmacological activities and therapeutic properties as anti-inflammatory, antiviral and nematocidal activities (Maheshwari et al. 2006; Cao et al. 2007). Moreover, curcumin is among the National Institutes of Health list of drugs being evaluated clinically as a chemopreventive drug in a variety of cancers (Kelloff et al. 2000; Bemis et al. 2006). Curcumin, a spice once relegated to the kitchen shelf, has moved into the clinic and may prove to be "Curecumin" (Goel et al. 2008). Moreover, Gupta et al. (2008) reported that topical instillation of aqueous extracts of curcumin showed potent anti-inflammatory activity against endotoxin-induced uveitis in rabbits. Previous studies have consistently shown that curcumin is relatively nontoxic, even at much high doses (Sharma et al. 2004).
Thus, the present study was aimed to investigate the possible protective effect of curcumin in preventing the renal changes caused by administration of cyclosporine in adult male rats. Oxidative stress in renal tissue samples was estimated by histological examination of morphological changes and the immunoexpression of GST as a marker of cellular antioxidant defense processes.

\section{Materials and Methods}

In the present study, twenty seven adult male albino rats (3-6 months) weighing 150-200 g were subjected to standard environmental conditions and allowed free access to normal rat chow diet and water ad libitum. One week after acclimatization rats were randomly divided into three equal groups (nine animals each). Group I (control group) was further subdivided into three equal subgroups, included animals that didn't receive any medication, animals that received a daily subcutaneous injection of olive oil (the vehicle of CsA) in a dose of $1 \mathrm{ml} / \mathrm{kg}$ body weight (b.w.) and subgroup included animals that received daily oral curcumin dissolved in distilled water by orogastric tube in a dose of $15 \mathrm{mg} / \mathrm{kg}$ b.w. Group II (CsA-treated group) included animals received a daily subcutanous injection of CsA dissolved in olive oil in a dose of $20 \mathrm{mg} / \mathrm{kg}$ b.w. (Tirkey et al. 2005). CsA (Sandimmun Neoral) obtained from Novartis Company in the form of soft gelatine capsules $(25 \mathrm{mg})$. The capsule was dissolved in olive oil. Group III (prophylactic group) included animals given curcumin (Sigma Chemical, USA) simultaneously with CsA. It has been dissolved in distilled water and given orally in a daily dose of $15 \mathrm{mg} / \mathrm{kg}$ b.w. by orogastric tube (Tirkey et al. 2005).

After 21 days, all the animals were anaesthetized using ether inhalation. Intracardial perfusion with $2.5 \%$ glutaraldehyde in $0.1 \mathrm{~mol} / \mathrm{l}$ cacodylate buffer was done. The kidneys were rapidly removed and opened to be processed for light microscope examination. The specimens were fixed in $10 \%$ buffered formalin for 24 hours to prepare paraffin blocks and thick sections $(5 \mu \mathrm{m})$ were obtained. For histological study, the sections were stained with haematoxylin and eosin, Periodic Acid Schiff's (PAS) and Masson's trichrome (Bancroft and Gamble 2002). For immunohistochemical study, the deparaffinized sections on charged slides were used for localization of GST enzyme using avidin-biotin-complex $(\mathrm{ABC})$ immunoperoxidase technique. The sections were incubated in hydrogen peroxide $0.1 \%$ for $30 \mathrm{~min}$ to block the endogenous peroxidase then incubated with the primary antibody. The primary antibody used was a rabbit polyclonal antibody (GST pi Ab-1; Lab Vision Corporation) which was diluted 1:400 and applied to the slides for 2 hours. Then, the slides were incubated with the secondary anti-rabbit antibodies uni- 
versal kits (Zymed) diluted 1 : 200 for $30 \mathrm{~min}$. Staining was completed by incubation with substrate chromogen called diamiobenzidine (DAB) which resulted in brown-colored precipitate at the antigen sites and Mayer's hematoxylin was used as counter stain. For negative control, the primary antibody was replaced by phosphate buffer solution. Quantitative morphometric measurements were achieved by using the Leica Qwin 500 Image analyzer computer system in the Department of Histology, Faculty of Medicine, Cairo University. The area of the positive GST immunoreactions was done within 10 fields for each animal at a total magnification $\times 100$. In each chosen field the cortical renal tubules and glomeruli were enclosed inside the standard measuring frame. The positively reactive GST areas were masked by a blue binary color to be measured. The total area (in \%) of positive GST immunoreaction in each specimen was measured and their mean values for each group were obtained. The data obtained were subjected to statistical analysis using ANOVA. Also, the optical density of GST immunoexpression was measured in the cytoplasm of tubules and glomeruli within 10 fields for each animal at a total magnification $\times 400$. Optical density was measured inside small measuring frames. The data obtained were subjected to statistical analysis using Kruskal-Wallis test. The level of significance $p<0.05$ was considered as statistically significant. Software Statistical package for the social sciences (SPSS) for Windows version 11 was used for all statistical calculation.

\section{Results}

\section{Histological results}

Light microscope examination of haematoxylin and eosin stained sections from kidneys of control animals (Group I) showed the renal cortex comprised renal corpuscles and tubules. The renal corpuscles were formed of glomerular tuft of capillaries and surrounded by Bowman's capsules with outer parietal layer of simple squamous epithelium and an inner visceral layer separated by Bowman's space. The convoluted tubules lined by cuboidal cells. The proximal ones had narrow irregular lumen; however, the distal tubules exhibited clear lumen (Fig. 1A). The collecting tubules of renal medulla were lined with cuboidal cells and surrounded by small blood vessels (Fig. 1B). In the sections from CsA-treated animals (Group II), the epithelial cells lining of the renal tubules showed darkly stained pyknotic nuclei and others had vacuolated cytoplasm. The renal corpuscles contained shrunken glomeruli with widening of their Bowman's spaces. Their Bowman's capsules were thickened and inflammatory cellular infiltrate was observed between some renal corpuscles (Fig. 1C). The collecting tubules of renal medulla exhibited wide lumen and their lining cells contained darkly stained pyknotic nuclei. Inflammatory cellular infiltrate was also noticed between the collecting tubules (Fig. 1D). The sections from animals taken curcumin concomitantly with CsA (Group III) showed preserved structure of renal corpuscles and tubules, however, few tubular darkly stained pyknotic nuclei were detected. Numerous blood vessels were also noticed (Fig. 1E). Collecting tubules revealed preserved structure and surrounded by congested blood vessels and few inflammatory cellular infiltrate (Fig. 1F).

Examination of PAS stained sections from kidneys of control animals (Group I) showed strong PAS positive reactions of the apical brush borders of proximal convoluted tubules and tubular basement membranes (Fig. 2A). The sections from CsA-treated animals (Group II) showed areas of ill-defined PAS reaction of apical brush borders and PAS positive granules in the cytoplasm of many tubular cells (Fig. 2B). The sections from animals taken curcumin concomitantly with CsA (Group III) showed positive PAS reaction of preserved apical brush borders of proximal convoluted tubules (Fig. 2C).

Examination of Masson's trichrome stained sections from kidneys of the control animals (Group I) showed few collagen fibers between the glomerular capillaries, tubules and around Bowman's capsule (Fig. 3A). While sections from CsA-treated animals (Group II) showed many collagen fibers between the renal tubules (Fig. 3B), the sections from animals taken curcumin concomitantly with CsA (Group III) showed less collagen fibers between the glomerular capillaries, renal tubules and around the Bowman's capsule (Fig. 3C), than in Group II.

\section{Immunohistochemical results}

The examination of kidney-sections of control animals (Group I) showed moderate GST immunoreaction in the cells of renal tubules and glomeruli (Fig. 4A). The sections from kidneys from CsA-treated animals (Group II) showed strong positive GST immunoreaction in the cells of many renal tubules and glomeruli (Figs. $4 \mathrm{~B}$ and $4 \mathrm{C}$ ). However, sections from animals taken curcumin concomitantly with CsA (Group III) showed moderate GST immunoreaction in the cytoplasm of tubular cells, few cells exhibited strong positive immunoreaction (Fig. 4D).

\section{Statistical analysis}

Statistical analysis of the optical density of the GST immunoreaction showed significant increase in Group II when compared with Group I or Group III. However, a non-significant increase in optical density of the GST immunoreaction was detected in the Group III when compared with Group I 
A

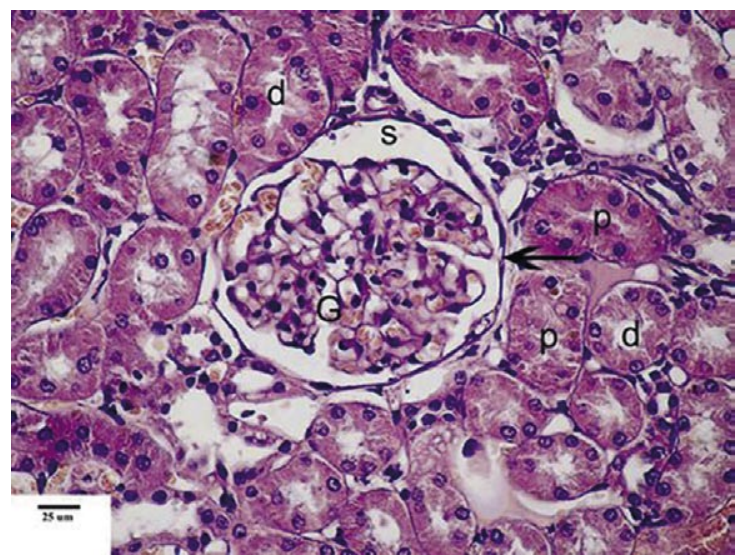

C

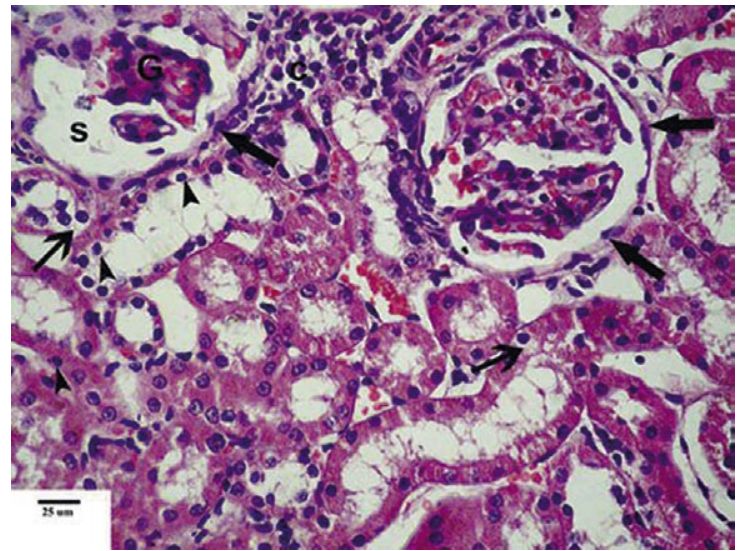

E

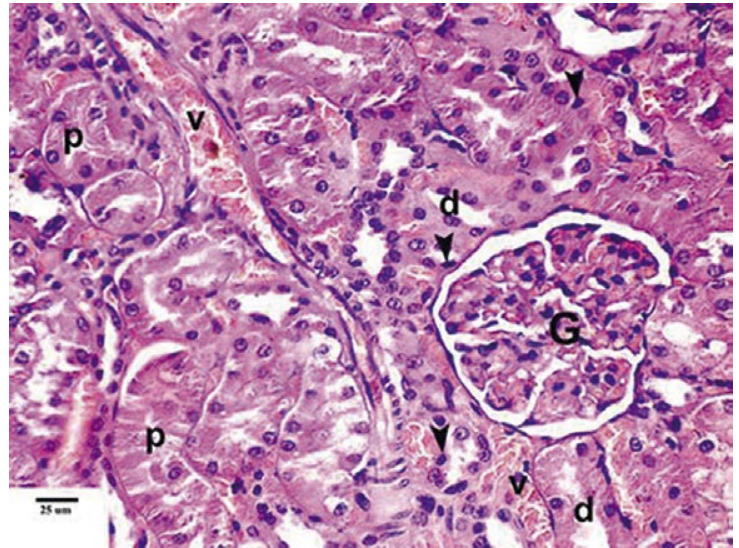

B

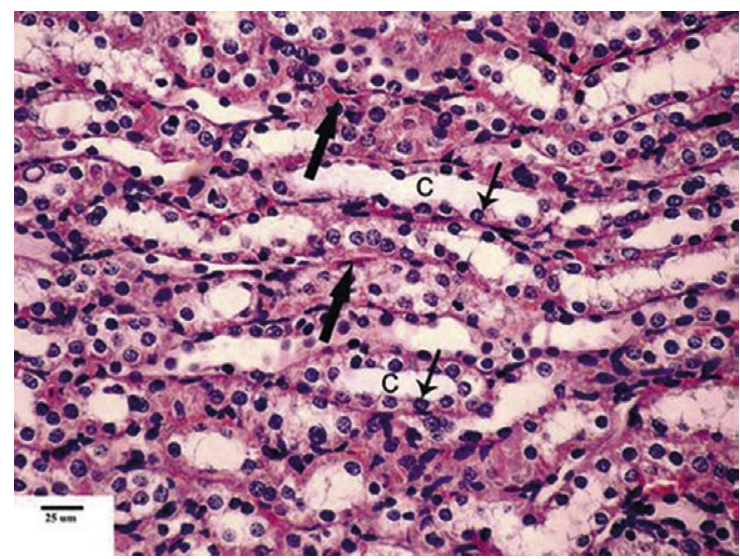

D

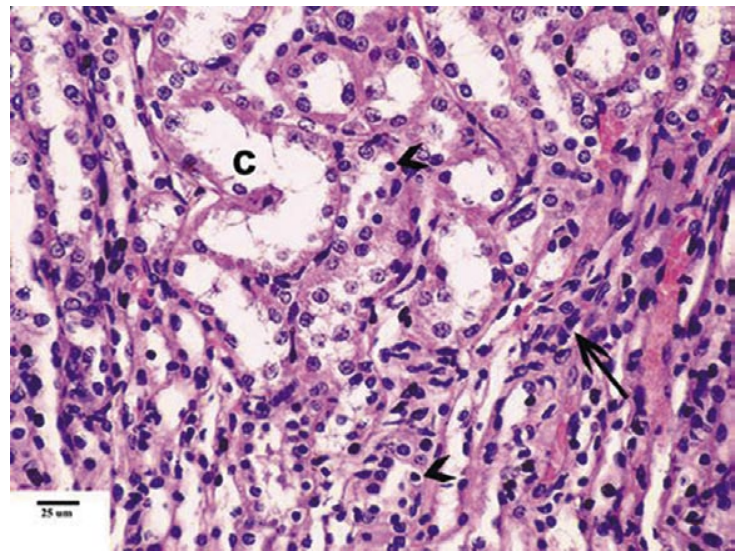

F

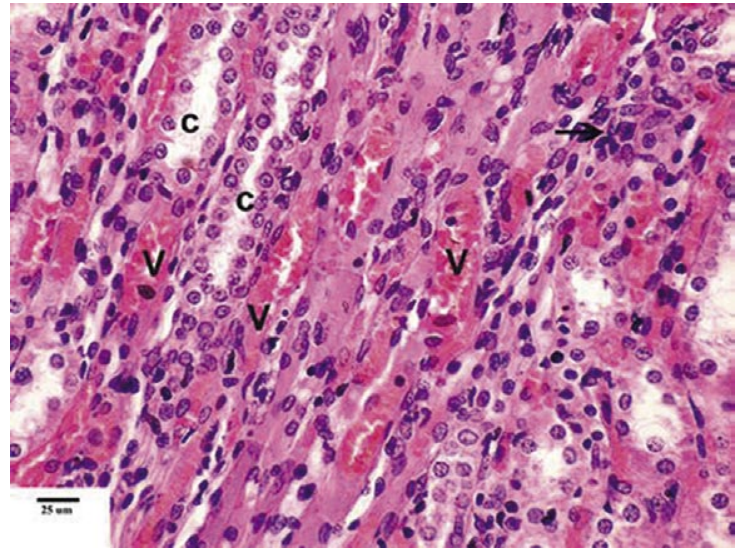

Figure 1. A. Section in the renal cortex of control albino rat showing a renal corpuscle with a glomerulus (G) surrounded by Bowman's capsule (arrow) and space (s). Proximal (p) and distal convoluted tubules (d) are also seen. B. Section in the renal medulla of control albino rat showing collecting tubules (c) lined by cuboidal cells (arrows) and surrounded by small blood vessels (thick arrows). C. Section in the renal cortex of a CsA-treated albino rat showing epithelial cells lining the renal tubules with darkly stained pyknotic nuclei (arrowheads) and vacuolated cytoplasm (arrows). A renal corpuscle with shrunken glomerulus (G) and widen Bowman's space (s) is seen. Thickened Bowman's capsule (thick arrows) and interstitial cellular infiltrate (c) are also noticed. D. Section in the renal medulla of CsA-treated albino rat showing collecting tubule with dilated lumen (c) and their lining cells appear with darkly stained pyknotic nuclei (arrow heads). Inflammatory cellular infiltration (arrow) is also noticed. E. Section in the renal cortex of curcumin and CsA-treated albino rat showing proximal (p), distal (d) tubules and renal corpuscles $(G)$ with preserved structure. Few cells lining the tubules appeared with darkly stained pyknotic nuclei (arrow heads). Numerous blood vessels (v) are also noticed. F. A photomicrograph of a section in the renal medulla of curcumin and CsA-treated albino rat showing collecting tubules (c) lined with cuboidal cells surrounded by congested blood vessels (V) and few cellular infiltration (arrows) (H\&E; Scalebar $=25 \mu \mathrm{m})$. 
A

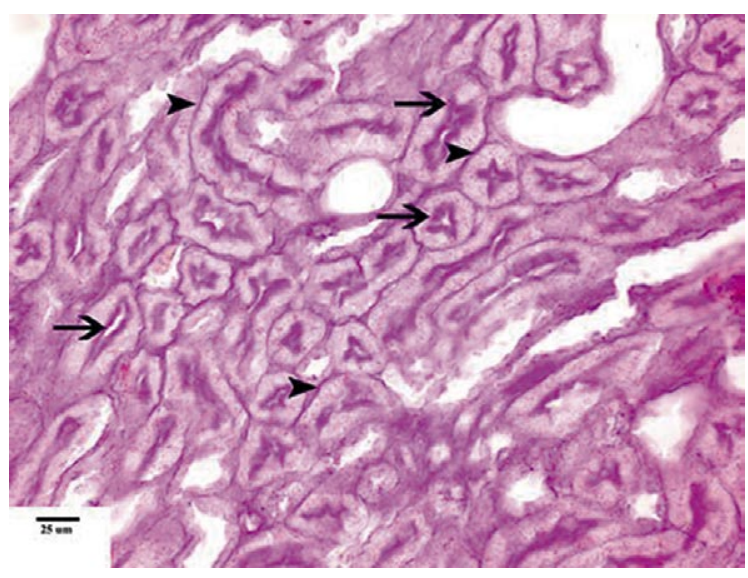

C

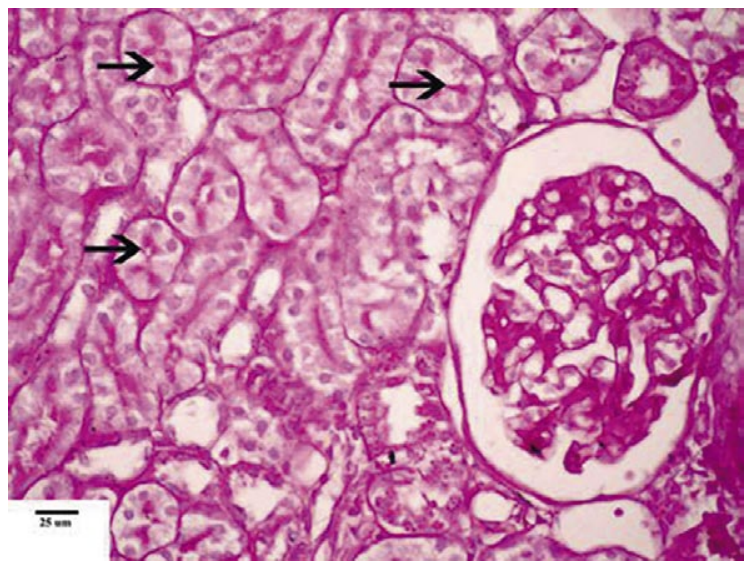

(Table 1). Statistical analysis of area of the GST immunoreaction among studied groups showed significant decrease in Group III when compared with Group II. However, a non significant increase in the area of the GST immunoreactive areas was detected in Group II when compared with Group I (Table 2).

\section{Discussion}

The kidney is the major target organ of CsA toxicity (Zhong et al. 1999). The renal damage induced by CsA is time and dose dependant (Khanna et al. 2004). Curcumin

\section{B}

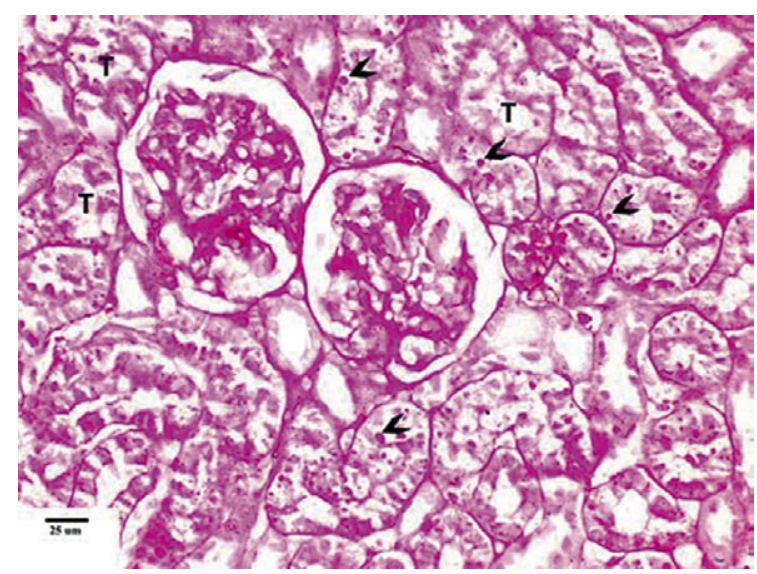

Figure 2. A. Section in the renal cortex of a control albino rat showing strong PAS positive reaction of the apical brush border (arrows) of proximal convoluted tubules and tubular basement membranes (arrowheads). B. Section in the renal cortex of CsA-treated albino rat showing ill-defined PAS reaction of brush borders of many renal tubules (T) and PAS positive bodies (arrow heads) in the cytoplasm of renal tubules. C. Section in the renal cortex of curcumin and CsA-treated albino rat showing PAS positive reaction of preserved apical brush borders (arrows) of the proximal convoluted tubules (PAS; Scalebar $=25 \mu \mathrm{m})$.

was reported to be a regulator of the expression of inflammatory enzymes, cytokines, adhesion molecules, and cell survival proteins by modulating the activation of various transcription factors (Goel et al. 2008).

In the current study, the cells lining renal tubules from CsA-treated animals contained vacuolated cytoplasm. The mechanism by which CsA caused these findings could be the prolonged vasoconstriction which induced a chronic relative ischemia, potentially responsible for cellular atrophy (Amore et al. 2000). In addition, Tirkey et al. (2005) mentioned that deterioration in membrane integrity was the marked cellular changes detected with CsA nephrotoxicity.

Table 1. Optical density (OD) of GST immunoreactivity among studied groups

\begin{tabular}{|c|c|c|c|c|}
\hline \multirow{2}{*}{ Group } & \multicolumn{2}{|c|}{ OD } & \multirow{2}{*}{ Median } & \multirow[b]{2}{*}{$p$} \\
\hline & Mean \pm SD & (Range) & & \\
\hline I (Control) & $0.53 \pm 0.03$ & $(0.50-0.61)$ & 0.52 & - \\
\hline $\mathrm{II}^{*}$ (CsA-treated ) & $0.58 \pm 0.02$ & $(0.55-0.61)$ & 0.57 & $0.0014^{*}$ \\
\hline III (Curcumin + CsA-treated) & $0.54 \pm 0.01$ & $(0.52-0.55)$ & 0.54 & $0.06(\mathrm{NS})$ \\
\hline
\end{tabular}

Kruskal-Wallis $=15.897{ }^{*}$ significant; SD, standard deviation; NS, non significant. 
A

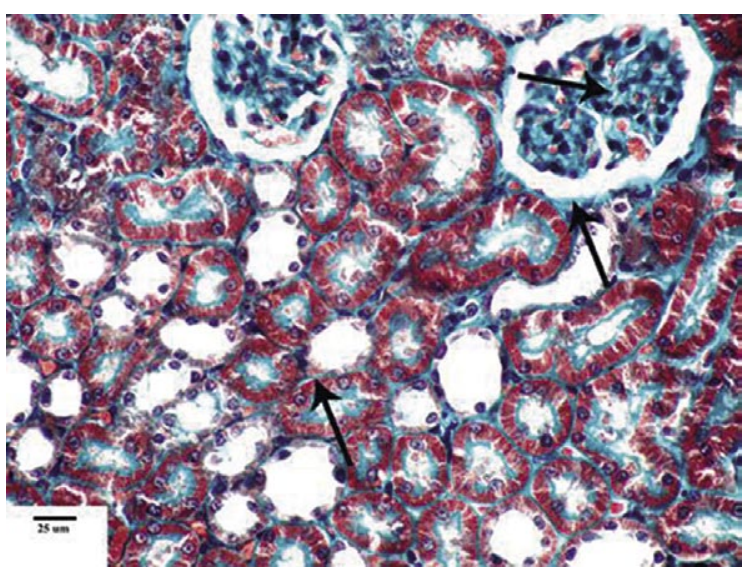

C

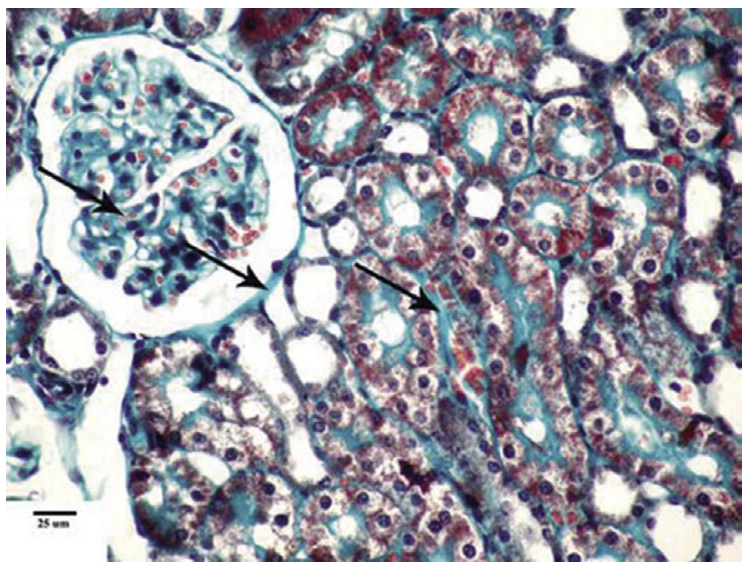

Also, examination of sections from CsA-treated animals showed undefined apical brush borders of some proximal tubules. Moreover, PAS positive granules were noticed in the cytoplasm of many tubular cells. These observations were in agreement with Kang et al. (2001). Li et al. (2003) and Khanna et al. (2004) detected tubular and vascular changes, including thickening of basement membranes and increased PAS staining of vascular structures in cyclosporine-treated animals. They added that afferent arteriolopathy was manifested by replacement of smooth muscle cells of afferent glomerular arteriole by a PAS-positive material.

The renal corpuscles of CsA-treated animals in the current work contained shrunken glomeruli with widening of their

\section{B}

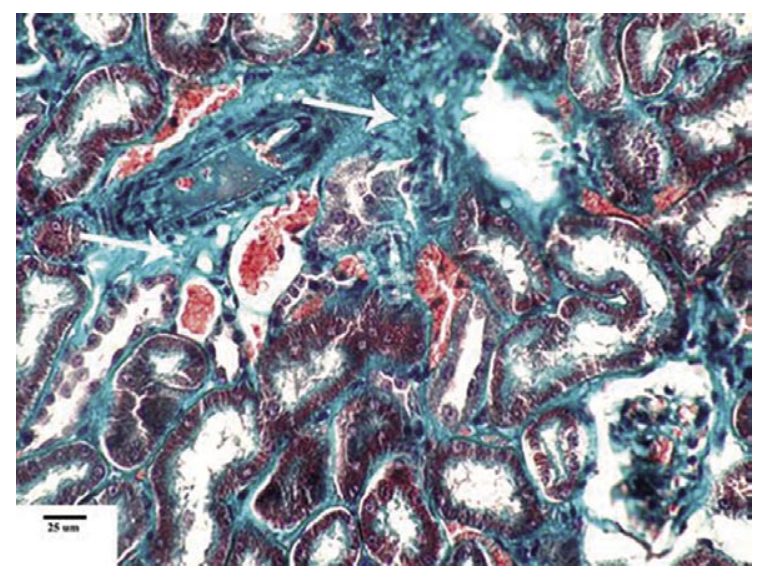

Figure 3. A. Section in the renal cortex of a control albino rat showing few collagen fibers (arrows) between the glomerular capillaries, renal tubules and around Bowman's capsule. B. Section in the renal cortex of CsA-treated albino rat showing many collagen fibers between the renal tubules (arrows). C. Section in the renal cortex of curcumin and CsA-treated albino rat showing few collagen fibers (arrows) between the renal tubules, glomerular capillaries and around Bowman's capsule (Masson's trichrome; Scalebar $=25 \mu \mathrm{m})$.

Bowmans spaces. These findings were in consistence with Amore et al. (2000) who reported that CsA induces apoptosis in mesangial, tubular, and endothelial cells in culture. CsA causes nephrotoxicity in the rat characterized by reduced glomerular filtration rates and increased serum creatinine (Thurman et al. 1997). Previous authors demonstrated that cyclosporine increases level of reactive oxygen metabolites in endothelial and mesangial cells indicating oxidative stress (Parra et al. 1998). These glomerular changes could be explained by glomerular thrombosis and vascular necrosis associated with cyclosporine toxicity in humans (Zarifian et al. 1999).

In the present work, darkly stained pyknotic nuclei were observed in the cells lining renal tubules. This finding was

Table 2. Comparison of area (in \%) of GST immunoreactivity among studied groups

\begin{tabular}{lrccc}
\hline Group & Mean \pm SD & Area (\%) & Median & \multicolumn{1}{c}{ (Range) } \\
\hline I (Control) & $37.9 \pm 9.6$ & $(19.80-45.64)$ & 41.21 & - \\
II (CsA-treated) & $41.3 \pm 9.9$ & $(23.64-52.73)$ & 43.13 & 0.55 NS \\
III (Curcumin + CsA-treated) & $25.98 \pm 6.1$ & $(17.05-36.86)$ & 25.58 & $0.004^{*}$ \\
\hline
\end{tabular}

ANOVA: $\mathrm{F}=8.497 ; \mathrm{P}=0.0016,{ }^{*}$ significant; $\mathrm{SD}$, standard deviation; NS, non significant. 
A

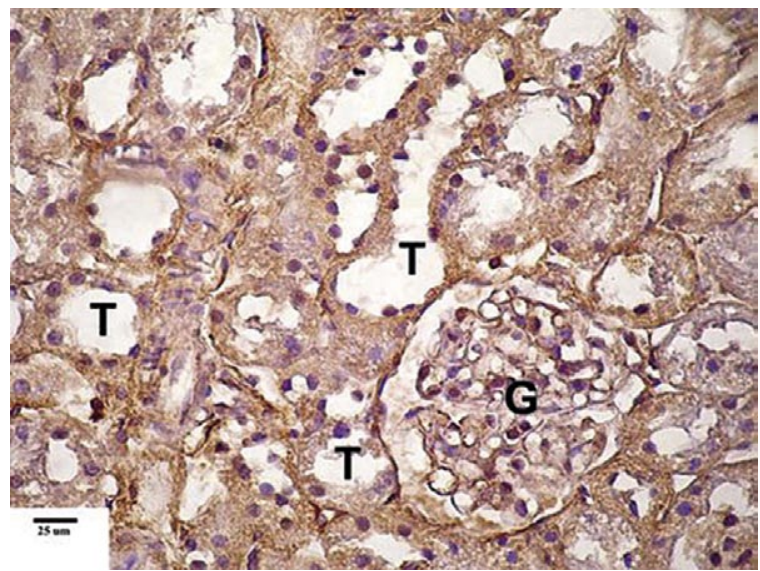

C

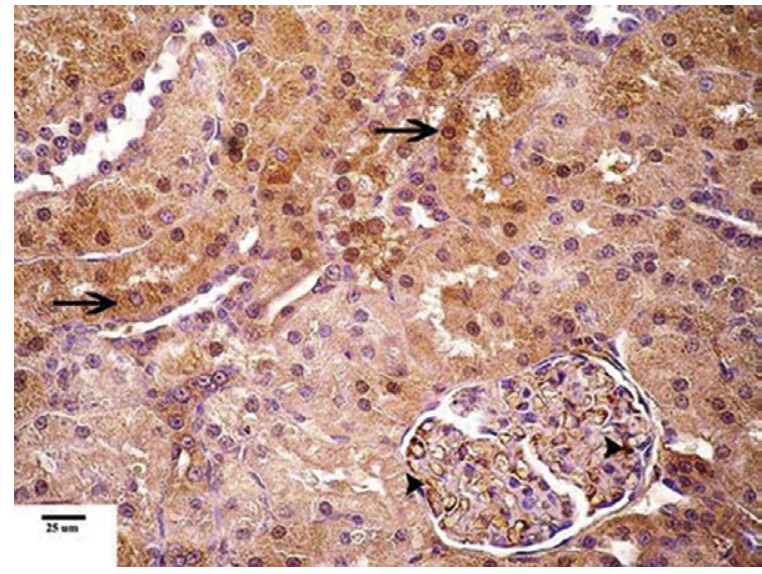

B

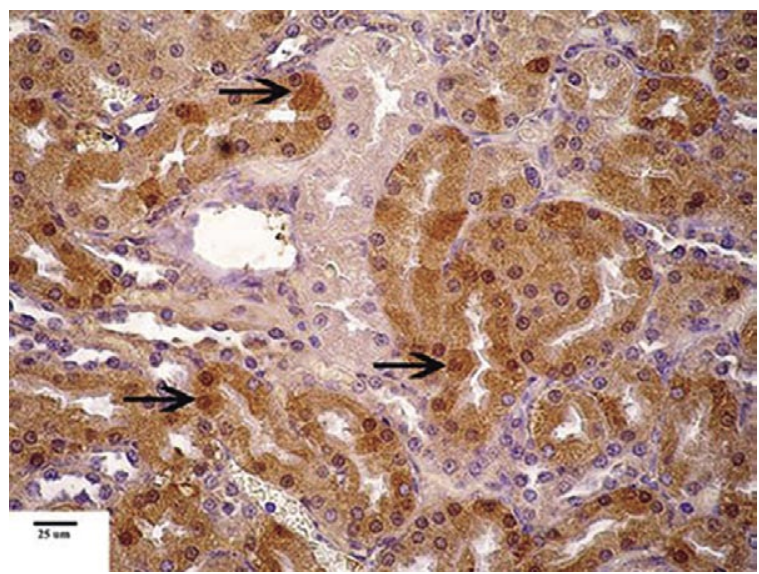

D

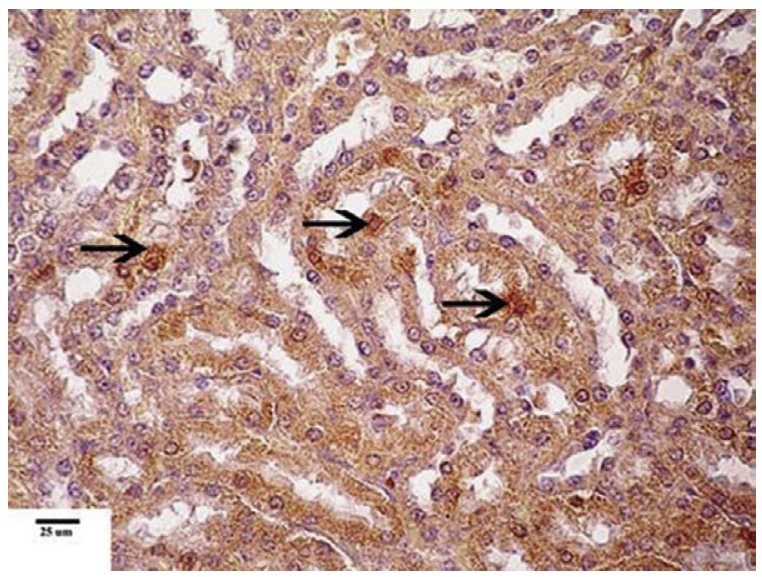

Figure 4. A. Section in the renal cortex of a control albino rat showing moderate positive immunoreaction for GST in the cytoplasm of the cells of the renal tubules $(\mathrm{T})$ and glomeruli $(\mathrm{G})$. B. Section in the renal cortex of CsA-treated albino rat showing strong positive immunoreaction for GST in the cells of many renal tubules (arrows). C. Section in the renal cortex of CsA-treated albino rat showing strong positive immunoreaction in the cytoplasm of many tubules (arrows) and glomerulus (arrow heads). D. Section in the renal cortex of curcumin and CsA-treated albino rat showing moderate GST immunoreaction in the cytoplasm of renal tubules. However, few cells appear with strong immunoreaction (arrows) (GST immunostaining; Scalebar $=25 \mu \mathrm{m}$ ).

in agreement with Han et al. (2006) who observed the presence of an increase in apoptotic cells in close association with the pathologic signs of CsA tubulo-interstitial damage. They attributed this fact to increase of expression in specific apoptotic genes. Lethally injured cells may die by either necrosis or apoptosis, depending on the severity of the injury (Nagata 1997). Examinations of sections from curcumin and CsA-treated animals (prophylactic group) showed preserved structure of renal corpuscles and tubules except few tubular darkly stained pyknotic nuclei. These observations were coincided with Tirkey and co-workers (2005). The protective effect of curcumin could be explained by Salvioli and co-workers (2007) who reported that curcumin can exert both pro- and anti-oxidant effects against apoptosis.

Numerous blood vessels were noticed in the sections from prophylactic group. Al-Sultan and Gameel (2004) also observed hyperaemia and mononuclear cell infiltration in parenchyma and portal areas in the liver of chicken received dietary supplement of turmeric (Curcuma longa) at different concentrations. Curcumin is a potent inhibitor of vascular smooth muscle cell functions and may play a critical role in regulating these events after vascular injury (Yang et al. 2006). In addition, curcumin can induce relaxation of isolated porcine coronary arteries (Xu et al. 2007).

Concerning interstitial cellular infiltration that was observed in CsA-treated group, Zhong et al. (2001) reported that CsA caused white blood cell infiltration. Also, infiltration of macrophages, and interstitial fibrosis were detected with CsA therapy at doses ranging from 25 to $50 \mathrm{mg} / \mathrm{kg}$ (Young et al. 1995). In contrast, Ying et al. (2003) reported that CsA is known to inhibit T-cell proliferation, induce apoptosis of CD4-positive T-cells and down regulate cytokine gene expression. Moreover, Tirkey et al. (2005) concluded 
that cyclosporine specifically and reversibly inhibits immunocompetent T-helper lymphocytes.

Many collagen fibers were observed between the renal tubules in the CsA-treated animals. Shihab et al. (1999) also observed that interstitial fibrosis caused by CsA consisted of matrix-rich expansion of the interstitium with distortion and collapse of the tubules and thickening of the tubular basement membranes. On the other hand, examination of sections from prophylactic group showed few collagen fibers between the renal tubules. Curcumin significantly attenuated interstitial macrophage influx and renal fibrosis (Kuwabara et al. 2006) and might also help to identify a new therapeutic pathway against hyperplasia of the synovial fibroblasts in rheumatoid arthritis (Park et al. 2007).

As regards GST immunoexpression, strong positive immunoreaction was observed in the cytoplasm of many tubules and glomeruli in the CsA-treated group. Glutathione plays a major role in detoxication of many reactive metabolites either by spontaneous conjugation or by GSTs (Hayes et al. 2005). GST enzyme detoxifies reactive oxygen species (which is induced by a variety of compounds including phenobarbital, antioxidants, active oxygen species and carcinogens). GST biosynthesis can be stimulated by a diverse range of biotic stress factors (e.g. pathogen invasion) and abiotic stress factors (e.g. heat shock, ozone, ethylene, heavy metals, and xenobiotic compounds), as well as by some inducers or chemoprotective substances (Manar et al. 2004). Resultant glutathione conjugates are less toxic than the parent compounds and can be stored in vacuoles or excreted from the biological system, helping the organism regain normal cellular functions and reducing the cellular toxicity of reactive parent or organic intermediates (Greulich et al. 2002). While examination of the kidney sections from prophylactic group showed moderate GST immunoreaction in the tubular cells, however, some cells showed strong reaction. These results were confirmed by significant decrease in the area of the GST immunoreaction in sections from prophylactic group when compared with that from CsA-treated and control groups. Joe et al. (2004) and Sandur et al. (2007) reported that curcumin exhibited anti-inflammatory, antiviral and proapoptotic effects and it is also considered as a potent scavenger of reactive oxygen and nitrogen species. Hence the exogenous antioxidant curcumin might compensate the need of the renal cells to the endogenous glutathione antioxidant. Yance and Tabachnik (2007) suggested a protective effect of curcumin against human cancer and cardiovascular risk due to the consumption of polyphenolic-rich herbs. They also added that phenolic antioxidants are known to stop lipid peroxidation of cell membranes, a prominent free radical chain reaction among unsaturated fatty acids that is carcinogenic by virtue of being both mutagenic and mitogenic. Some of these activities are responsible for the ability of curcumin to protect DNA against free radical-induced damage and to protect biological cells against various toxins (Sahu and Washington 1992).

\section{Conclusion}

The protective effect of curcumin against cyclosporineinduced nephrotoxicity in rats was proven based on the study of histological changes and GST immunoexpression. So, curcumin may have possible therapeutic applications in CsA-treated patients.

\section{References}

Al-Sultan S. I., Gameel A. A. (2004): Histopathological changes in the livers of broiler chicken supplemented with turmeric (Curcuma longa). Int. J. Poult. Sci. 3, 333-336; doi:10.3923/ijps.2004.333.336

Amore A., Emancipator S. N., Cirina P., Conti G., Ricotti E., Bagheri N., Coppo R. (2000): Nitric oxide mediates cyclosporineinduced apoptosis in cultured renal cells. Kidney Int. 57, 1549-1559; doi:10.1046/j.1523-1755.2000.00999.x

Bancroft J., Gamble A. (2002): Theory and Practice of Histological Techniques. 5th ed., Churchil Livingstone, New York

Bemis D. L., Katz A. E., Buttyan R. (2006): Clinical trials of natural products as chemopreventive agents for prostate cancer. Expert Opin. Investig. Drugs 15, 1191-1200; doi:10.1517/ 13543784.15.10.1191

Cao J. Liu Y., Jia L., Zhou H. M., Kong Y., Yang G., Jiang L. P., Li Q. J., Zhong L. F. (2007): Curcumin induces apoptosis through mitochondrial hyperpolarization and mtDNA damage in human hepatoma G2 cells. Free Radic. Biol. Med. 43, 968-975; doi:10.1016/j.freeradbiomed.2007.06.006

Faulds D., Goa P. L., Benfield P. (1993): Cyclosporine A: a review of its pharmacodynamic and pharmacokinetic properties, and therapeutic use in immunoregulatory disorders. Drugs 45, 953-1040; doi:10.2165/00003495-199345060-00007

Goel A., Kunnumakkara A. B., Aggarwal B. B. (2008): Curcumin as „Curecumin“: from kitchen to clinic. Biochem. Pharmacol. 75, 787-809; doi:10.1016/j.bcp.2007.08.016

Greulich K., Hoque E., Pflugmacher S. (2002): Uptake, metabolism, and effects on detoxification enzymes of isoproturon in spawn and tadpoles of amphibians. Ecotoxicol. Environ. Saf. 52, 256-266; doi:10.1006/eesa.2002.2182

Gupta S. K., Agarwal R., Srivastava S., Agarwal P., Agrawal S. S., Saxena R., Galpalli N. (2008): The anti-inflammatory effects of Curcuma longa and Berberis aristata in endotoxin-induced uveitis in rabbits. Invest. Ophthalmol. Vis. Sci. 49, 4036-4040; doi:10.1167/iovs.07-1186

Han S. Y., Chang E. J., Choi H. J., Kwak C. S., Park S. B., Kim H. C., Mun K. C. (2006): Apoptosis by cyclosporine in mesangial cells. Transplant. Proc. 38, 2244-2246; doi:10.1016/j.tran sproceed.2006.06.029

Hayes J. D., Flanagan J. U., Jowsey I. R. (2005): Glutathione transferases. Annu. Rev. Pharmacol. Toxicol. 45, 51-88; doi:10.1146/annurev.pharmtox.45.120403.095857 
Joe B., Vijaykumar M., Lokesh B. R. (2004): Biological properties of curcumin-cellular and molecular mechanisms of action. Crit. Rev. Food Sci. Nutr. 44, 97-111; doi:10.1080/ 10408690490424702

Kang D., Kim Y. G, Andoh T. F., Gordon K. L., Suga S., Mazzali M., Jefferson J. A., Hughes J., Bennett W., Schreiner G. F., Johnson R. J. (2001): Post-cyclosporine-mediated hypertension and nephropathy: amelioration by vascular endothelial growth factor. Am. J. Physiol. Renal Physiol. 280, F727-736

Kelloff G. J., Crowell J. A., Steele V. E., Lubet R. A., Malone W. A., Boone C. W., Kopelovich L., Hawk E. T., Lieberman R., Lawrence J. A., Ali I., Viner J. L., Sigman C. C. (2000): Progress in cancer chemoprevention: development of diet-derived chemopreventive agents. J. Nutr. 130, S467-471

Khanna A. K., Plummer M. S., Hilton G., Pieper G. M., Ledbetter S. (2004): Anti-transforming growth factor antibody at low but not high doses limits cyclosporine-mediated nephrotoxicity without altering rat cardiac allograft survival: potential of therapeutic applications. Circulation 110, 3822-3829; doi:10.1161/01.CIR.0000150400.15354.7D

Kopáni M., Celec P., Danisovic L., Michalka P., Biró C. (2006): Oxidative stress and electron spin resonance. Clin. Chim. Acta 364, 61-66; doi:10.1016/j.cca.2005.05.016

Kuwabara N., Tamada S., Iwai T., Teramoto K., Kaneda N., Yukimura T., Nakatani T., Miura K. (2006): Attenuation of renal fibrosis by curcumin in rat obstructive nephropathy. Urology 67, 440-446; doi:10.1016/j.urology.2005.09.028

Li C., Yang C. W., Kim W. Y., Jung J. Y., Cha J. H., Kim Y. S., Kim J., Bennett W. M., Bang B. K. (2003): Reversibility of chronic cyclosporine nephropathy in rats after withdrawal of cyclosporine. Am. J. Physiol. Renal Physiol. 284, F389-398

Maheshwari R. K., Singh A. K., Gaddipati J., Srimal R. C. (2006): Multiple biological activities of curcumin: a short review. Life Sci. 78, 2081-2087; doi:10.1016/j.lfs.2005.12.007

Manar M. H., Brown M. R., Gauthier T. W., Brown L. A. (2004): Association of glutathione-S-transferase-P1 (GST-P1) polymorphisms with bronchopulmonary dysplasia. J. Perinatol. 24, 30-35; doi:10.1038/sj.jp.7211020

Nagata S. (1997): Apoptosis by death factor. Cell 88, 355-365; doi:10.1016/S0092-8674(00)81874-7

Park C., Moon D. O., Choi I. W., Choi B. T., Nam T. J., Rhu C. H., Kwon T. K., Lee W. H., Kim G. Y., Choi Y. H. (2007): Curcumin induces apoptosis and inhibits prostaglandin $\mathrm{E}(2)$ production in synovial fibroblasts of patients with rheumatoid arthritis. Int. J. Mol. Med. 20, 365-372

Park H. J., Lee K. S., Cho S. H., Kong K. H. (2001): Functional studies of cysteine residues in human glutathion-S-transferase P1-1 by site-directed mutagenesis. Bull. Korean Chem. Soc. 22, 77-82

Parra T., de Arriba G., Conejo J. R., Cantero M., Arribas I., Rodríquez-Puyol D., Rodriguez-Puyol M., Carballo F. (1998): Cyclosporine increases local glomerular synthesis of reactive oxygen species in rats: effect of vitamin $\mathrm{E}$ on cyclosporine nephrotoxicity. Transplantation 66, 13251329; doi:10.1097/00007890-199811270-00011
Rahman I., MacNee W. (1999): Lung glutathione and oxidative stress: implications in cigarette smoke-induced airway disease. Am. J. Physiol. Lung Cell Mol. Physiol. 277, L1067-1088

Sahu S. C., Washington M. C. (1992): Effect of ascorbic acid and Curcumin on quercetin-induced nuclear DNA damage, lipid peroxidation and protein degradation. Cancer Lett. 63, 237-241; doi:10.1016/0304-3835(92)90266-X

Salvioli S., Sikora E., Cooper E. L., Franceschi C. (2007): Curcumin in cell death processes: a challenge for CAM of age-related pathologies. Evid. Based Complement. Alternat. Med. 4, 181-190; doi:10.1093/ecam/nem043

Sandur S. K., Ichikawa H., Pandey M. K., Kunnumakkara A. B., Sung B., Sethi G., Aggarwal B. B. (2007): Role of prooxidants and antioxidants in the anti-inflammatory and apoptotic effects of curcumin (diferuloylmethane). Free Radic. Biol. Med. 43, 568-580; doi:10.1016/j.freeradbio med.2007.05.009

Sharma R. A., Euden S. A., Platton S. L., Cooke D. N., Shafayat A., Hewitt H. R., Marczylo T. H., Morgan B., Hemingway D., Plummer S. M., Pirmohamed M., Gescher A. J., Steward W. P. (2004): Phase I clinical trial of oral curcumin: biomarkers of systemic activity and compliance. Clin. Cancer Res. 10, 6847-6854; doi:10.1158/1078-0432. CCR-04-0744

Shihab F. S., Andoh T. F., Tanner A. M., Yi H., Bennett W. M. (1999): Expression of apoptosis regulatory genes in chronic cyclosporine nephrotoxicity favors apoptosis. Kidney Int. 56, 2147-2159; doi:10.1046/j.1523-1755.1999.00794.x

Sivonová M., Zitnanová I., Hlincíková L., Skodácek I., Trebatická J., Duracková Z. (2004): Oxidative stress in university students during examinations. Stress 7, 183-188; doi:10.1080/10253890400012685

Sivonová M., Zitnanová I., Horáková L., Strosová M., Muchová J., Balgavý P., Dobrota D., Duracková Z. (2006): The combined effect of pycnogenol with ascorbic acid and trolox on the oxidation of lipids and proteins. Gen. Physiol. Biophys. 25, 379-396

Thurman R. G., Zhong Z., von Frankenberg M. V., Stachlewitz R. F., Bunzendahl H. (1997): Prevention of cyclosporineinduced nephrotoxicity with dietary glycine. Transplantation 63, 1661-1667; doi:10.1097/00007890-19970615000021

Tirkey N., Kaur G., Vij G., Chopra K. (2005): Curcumin, a diferuloylmethane, attenuates cyclosporine-induced renal dysfunction and oxidative stress in rat kidneys. BMC Pharmacol. 5:15; doi:10.1186/1471-2210-5-15

Weis M., Kopáni M., Michalka P., Biró C., Celec P., Danisovic L., Jakubovský J. (2005): Conformation study of the membrane models by the Maxwell displacement current technique and oxidative stress. J. Biochem. Biophys. Methods 65, 81-87; doi:10.1016/j.jbbm.2005.10.005

Xu P., Long Y., Dai F., Liu Z. L. (2007): The relaxant effect of curcumin on porcine coronary arterial ring segments. Vascul. Pharmacol. 47, 25-30; doi:10.1016/j.vph.2007.03.003

Yang X., Thomas D. P., Zhang X., Culver B. W., Alexander B. M., Murdoch W. J., Rao M. N., Tulis D. A., Ren J., Sreejayan N. (2006): Curcumin inhibits platelet-derived growth 
factor-stimulated vascular smooth muscle cell function and injury-induced neointima formation. Arterioscler. Thromb. Vasc. Biol. 26, 85-90; doi:10.1161/01. ATV.0000191635.00744.b6

Ying S., Khan L. N., Meng Q., Barnes N. C., Kay A. B. (2003): Cyclosporine A, apoptosis of BAL T-cells and expression of Bcl-2 in asthmatics. Eur. Respir. J. 22, 207-212; doi:10.1183/09031936.03.00098902

Young B. A., Burdmann E. A., Johnson R. J., Alpers C. E., Giachelli C. M., Eng E., Andoh T., Bennett W. M., Couser W. G. (1995): Cellular proliferation and macrophage influx precedes interstitial fibrosis in cyclosporine nephrotoxicity. Kidney Int. 48, 439-448; doi:10.1038/ki.1995.312

Zarifian A., Meleg-Smith S., O'donovan R., Tesi R. J., Batuman V. (1999): Cyclosporine-associated thrombotic microan- giopathy in renal allografts. Kidney Int. 55, 2457-2466; doi:10.1046/j.1523-1755.1999.00492.x

Zhong Z., Connor H. D., Yin M., Moss N., Mason R. P., Bunzendahl H., Forman D. T., Thurman R. G. (1999): Dietary glycine and renal denervation prevents cyclosporine A-induced hydroxyl radical production in rat kidney. Mol. Pharmacol. 56, 455-463

Zhong Z., Connor H. D., Yin M., Wheeler M. D., Mason R. P., Thurman R. G. (2001). Viral delivery of superoxide dismutase gene reduces cyclosporine A-induced nephrotoxicity. Kidney Int. 59, 1397-1404; doi:10.1046/j.15231755.2001.0590041397.x

Received: February 16, 2009

Final version accepted: November 4, 2009 